\title{
THE PRESENT VALUE OF A SERIES OF CASHFLOWS : CONVERGENCE IN A RANDOM ENVIRONMENT
}

\author{
Andrew J. G. CAIRnS \\ Heriot-Watt University, Edinburgh, $U K^{1}$
}

\begin{abstract}
The present paper considers the present value, $Z(t)$, of a series of cashflows up to some time $t$. More specifically, the cashflows and the interest rate process will often be stochastic and not necessarily independent of one another or through time. We discuss under what circumstances $Z(t)$ will converge almost surely to some finite value as $t \rightarrow \infty$. This problem has previously been considered by DUFRESNE (1990) who provided a sufficient condition for almost sure convergence of $Z(t)$ (the Root Test) and then proceeded to consider some specific examples of such processes. Here, we develop Dufresne's work and show that the sufficient condition for convergence can be proved to hold for quite a general class of model which includes the growing number of Office Models with stochastic cashflows.
\end{abstract}

\section{KEYWORDS}

Stochastic discounting; cashflow models; almost sure convergence; office model.

\section{INTRODUCTION}

Suppose $\delta(t)$ is the constant force of interest during the period $[t-1, t)$, so that an investment of 1 at time $t-1$ will accumulate to $\exp \delta(t)$ at time $t$. The present value at time 0 of 1 due at time $t$ is then

$$
V(t)=\exp \left(-\sum_{s=1}^{t} \delta(s)\right)=\prod_{s=1}^{t} v_{s}
$$

where $v_{s}=\exp (-\delta(s))$ is the discount factor for year $s$.

The present value of a series of cashflows $C(1)$ at time $1, C(2)$ at time $2, \ldots, C(t)$ at time $t$ is therefore

$$
Z(t)=\sum_{s=1}^{t} V(s) C(s)=\sum_{s=1}^{t} v_{1} \ldots v_{s} C(s)
$$

\footnotetext{
${ }^{1}$ Address for correspondence: Andrew J.G. Cairns, Department of Actuarial Mathematics and Statistics, Heriot-Watt University, Riccarton, Edinburgh, EHI4 4AS, UK.

ASTIN BULLETIN, 1995, Vol. 25, No. 2, 81-94
} 
Such a process has been considered by DUFRESNE (1990) and AEBI et al. (1994) from the financial point of view and by VERVAAT (1979) and BRANDT (1986) from the mathematical point of view. All of these works consider the special case where $\{\delta(t)\}_{t=1}^{\infty}$ and $\{C(t)\}_{t=1}^{\infty}$ are independent and identically distributed and independent of one another. DUFRESNE (1990) considers the convergence of $Z(t)$ as $t$ tends to infinity and its limiting distribution when the distributions of $\delta(t)$ and $C(t)$ are known. AEBI et al. (1994) show how Bootstrap methodology can be used to estimate the limiting distribution when a limited number of past observations of $\delta(t)$ and $C(t)$ are available.

DUFRESNE (1990) also considers more general models and provides sufficient conditions for almost sure convergence of $Z(t)$. In this paper we consider a number of specific examples for the process $Z(t)$ and it is demonstrated that Dufresne's conditions hold for quite a wide class of models.

In this paper we will restrict ourselves to discrete time models. However, the results described here also hold for the continuous time models for $\delta(t)$ described by PARKer (1993, 1994d) and Norberg and MøLler (1994).

De SchepPer, Tuenen and GoOvaerts (1994) consider the present value of annuities and of a perpetuity payable continuously. Using Laplace transforms they show that the perpetuity has an inverse Gamma distribution, matching the results of DUFRESNE (1990).

\section{CONDITIONS FOR CONVERGENCE OF $Z(t)$}

The principal result provided by DUFRESNE (1990) giving a sufficient condition for the almost sure convergence of $Z(t)$ is the Root Test:

Theorem 1 (Root Test, for example, see Dufresne, 1990)

If

$$
\limsup _{t \rightarrow \infty}|V(t) C(t)|^{1 / t}<1 \text { almost surely }
$$

then $Z(t)$ converges almost surely to some finite limit as $t$ tends to infinity.

Now, trivially, this is equivalent to the condition

$$
\begin{aligned}
& \limsup _{t \rightarrow \infty} \frac{1}{t}\left\{\log |C(t)|+\sum_{s=1}^{t} \log v_{s}\right\}<0 \text { almost surely } \\
\Leftrightarrow & \limsup _{t \rightarrow \infty} \frac{1}{t}\left\{\log |C(t)|-\sum_{s=1}^{t} \delta(s)\right\}<0 \text { almost surely }
\end{aligned}
$$

We therefore have the following 


\section{Corollary 2}

If the force of interest process $\{\delta(t)\}_{t=1}^{\infty}$ is ergodic with $\frac{1}{t} \sum_{s=1}^{t} \delta(s) \rightarrow \delta=E[\delta()$. almost surely, and if

$$
\limsup _{t \rightarrow \infty} \frac{1}{t} \log |C(t)|=\rho \in[-\infty,+\infty)
$$

where $\rho-\delta<0$ then $Z(t)$ converges almost surely to some finite limit as $t$ tends to infinity.

[The condition that $\rho<\delta$ means that the cashflow process, $C(t)$, must grow more slowly than the accumulation process $1 / V(t)$. Consider the trivial case of a perpetuity where $C(t)=\exp \rho t$ and $V(t)=\exp -\delta t$ are both deterministic. Then $Z(t)$ converges if and only if $\rho<\delta$.]

Corollary 2 extends Dufresne's subsequent development by allowing the process $C(t)$ :

- to depend on the force of interest process;

- to be non-ergodic and, in particular, to include inflationary growth and growth in the underlying number of policies.

[A rigorous definition of ergodicity is given by KARLIN and TAYLOR (1975). If a process $X(t)$ is known to be ergodic then the following results hold:

$$
\begin{gathered}
\lim _{n \rightarrow \infty} \frac{1}{n} \sum_{t=1}^{n} X(t)=\mu \\
\lim _{n \rightarrow \infty} \frac{1}{n} \sum_{t=1}^{n}\left[X(t)-\bar{X}_{n}\right]^{2}=\sigma^{2} \text { where } \bar{X}_{n}=\frac{1}{n} \sum_{t=1}^{n} X(t) \\
\text { and if } I_{a, b}(x)= \begin{cases}1 & \text { if } a<x \leq b \\
0 & \text { otherwise }\end{cases} \\
\lim _{n \rightarrow \infty} \frac{1}{n} \sum_{t=1}^{n} I_{a, b}[X(t)]=F(b)-F(a)
\end{gathered}
$$

where $\mu$ and $\sigma^{2}$ are the unconditional mean and variance of $X(t)$ and $F(x)$ is the unconditional cumulative distribution function of $X(t)$.]

\section{THE INTEREST RATE PROCESS}

Before we concentrate on the cashflow process, it is worth discussing briefly some interest rate processes.

The ergodic condition for the force of interest process is not particularly onerous, and encompasses most of the widely used stochastic investment models. 
- Independent and identically distributed returns : for example, WatERs (1978), Dufresne (1990), Papachristou and Waters (1991), Parker (1993, 1994d) and AEBI et al. (1994) give but a few examples.

- Simple autoregressive models for the rate of return, such as the $A R(1)$ time series model, and the Ornstein-Uhlenbeck process: for example, DHAENE (1989, 1992), PARKer (1993, 1994a,b,c,d) and Norberg and Møller (1994).

- Models with several asset classes: for example, WILKIE (1986, 1987, 1992, 1994, 1995).

- Models for the term structure of interest rates: for example, BoYLE (1978, 1980), Brennan and Schwarz (1979), Albrecht (1985), CoX, Ingersoll and Ross (1985), BEeckMAN and Shiu (1988), Heath, Jarrow and Morton (1990, 1992), SERCu (1991) and LongstafF and SCHWARZ (1992).

- Autoregressive Conditionally Heteroscedastic (ARCH) models: for example, see Wilkie (1995, Appendix D).

Some non-ergodic models may still admit convergence but it is worth discussing some special cases which may be considered to be inappropriate.

First, DufResne (1990, Proposition 3.2.1) shows that $Z(t)$ will converge if $\left\{v_{s}\right\}$ is stationary and ergodic and $\operatorname{Pr}\left(v_{s}=0\right)>0$. This second condition is equivalent to $\operatorname{Pr}(\delta(s)=\infty)>0$ which does not seem appropriate.

Second, some authors (for example, DhaENe, 1989, 1992; Dufresne, 1990, and PARKER, 1993) consider non-stationary models for the force of interest process. One of two things may happen.

- The process may have positive drift, so that $\delta(t)$ tends to infinity almost surely (again unrealistic).

- The process may have no drift but unbounded variance. Examples of this are random walk models of the form $\delta(t+1)=\delta(t)+\varepsilon(t+1)$ where the $\varepsilon$ are independent and identically distributed zero mean random variables, and, more generally, $\operatorname{ARIMA}(p, d, q)$ models $(d \geq 1)$. With such processes it is often easy to show (for example, see Dufresne, 1990, Proposition 4.4.4) that

$$
\limsup _{t \rightarrow \infty}|Z(t)|=\infty \text {. }
$$

Figures 1 and 2 demonstrate the problem. For many years $\delta(t)$ may remain positive (Figure 1). The process $Z(t)$ (Figure 2) may then give one the impression that it is converging and that it is safe to use $Z(50)$ or $Z(100)$, say, as an approximation to $Z(\infty)$. After a while, though, $\delta(t)$ takes a long excursion below zero and $Z(t)$ shoots off upwards.

\section{THE CASHFLOW PROCESS}

Corollary 2 provided us with a condition for the behaviour of $C(t)$ as $t$ tends to infinity. We now consider this in more detail and provide the following lemma which will allow us to satisfy the conditions in Corollary 2. 


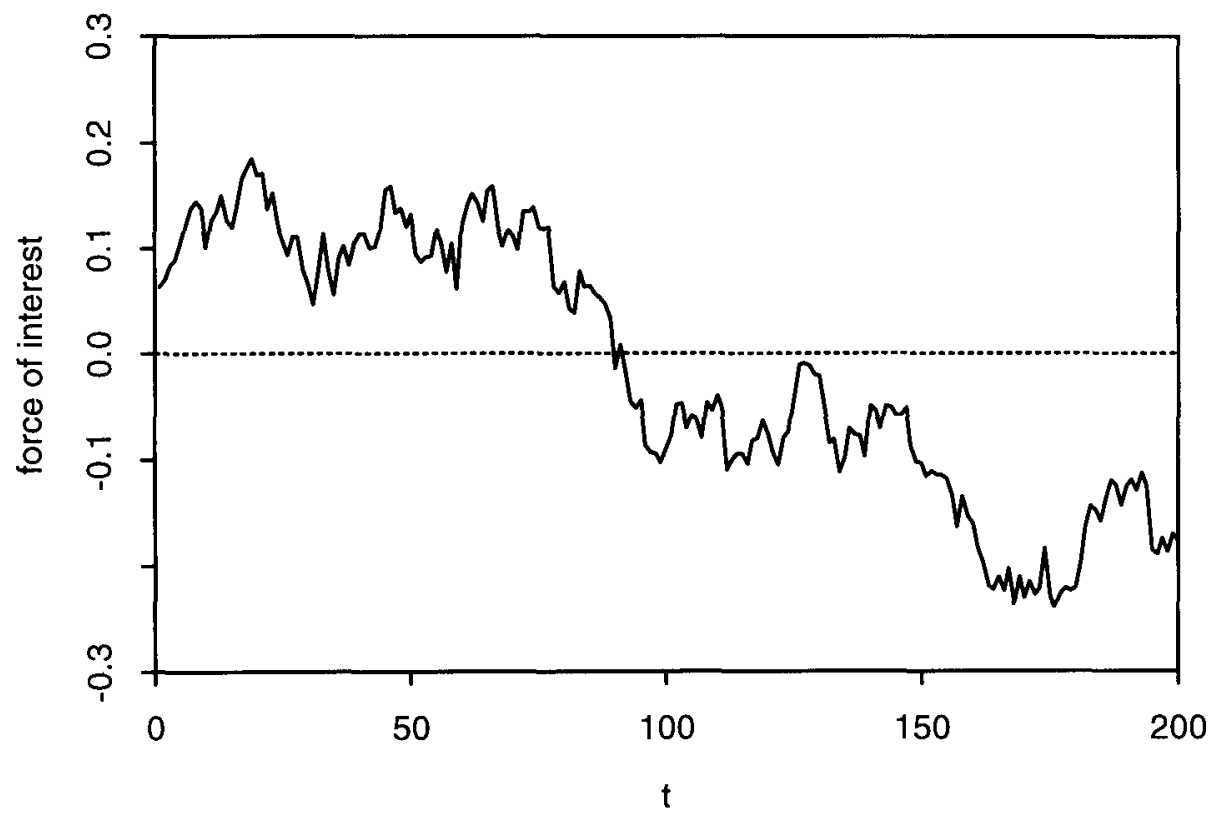

FigURE 1. Sample path of a random walk interest rate process, $\delta(t)$.

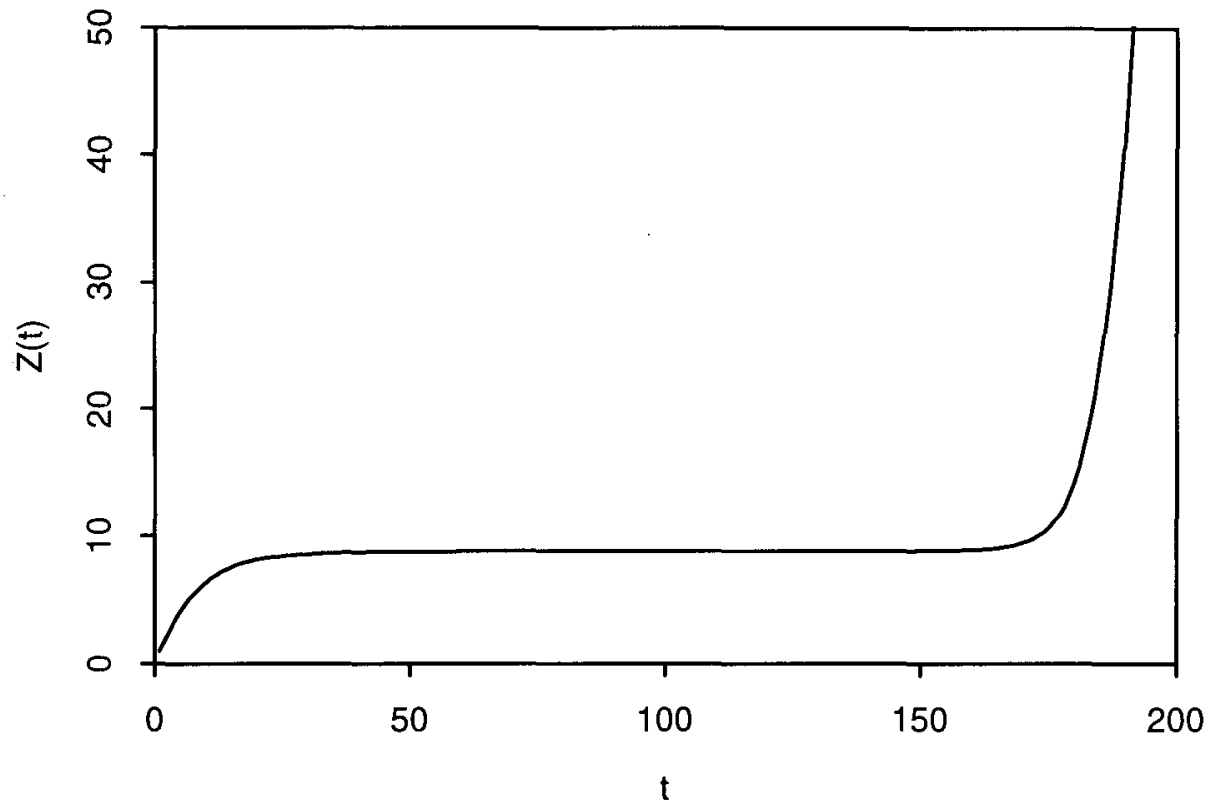

FIGURE 2. $Z(t)$ appears to converge initially but eventually diverges. 


\section{Lemma 3}

Suppose that there exists a deterministic sequence a $(t)$ converging to zero as $t$ tends to infinity such that

$$
\sum_{t=1}^{\infty} \operatorname{Pr}\left(\frac{1}{t} \log |C(t)| \geq \rho+a(t)\right)<\infty
$$

Then

$$
\limsup _{t \rightarrow \infty} \frac{1}{t} \log |C(t)| \leq \rho \text { almost surely. }
$$

\section{Proof}

$$
\begin{gathered}
\text { Let } E_{t}=\left\{t^{-1} \log |C(t)| \geq \rho+a(t)\right\} . \text { Then } \\
\qquad \sum_{t=1}^{\infty} \operatorname{Pr}\left(E_{t}\right)<\infty \\
\Rightarrow \operatorname{Pr}\left(\limsup _{t \rightarrow \infty} E_{t}\right)=0 \text { by the first Borel-Cantelli Lemma } \\
\quad \text { (for example, see WILLiaMs, 1991, Section 2.7) } \\
\Rightarrow \limsup _{t \rightarrow \infty} \frac{1}{t} \log |C(t)| \leq \rho \text { almost surely. }
\end{gathered}
$$

Lemma 3 provides quite a weak condition on the cashflow process : cashflows need not be independent; and the tails of the cashflow distributions can be quite fat. In particular if we suppose that $\mu(t)=E|C(t)|$ then we are able to prove the following theorem which provides us with a relatively easy method for proving the convergence of $t^{-1} \log |C(t)|$.

\section{Theorem 4}

$$
\begin{aligned}
& \text { Let } \rho_{0}=\inf \left\{\rho: \limsup _{t \rightarrow \infty} e^{-\rho_{t}} \mu(t)<\infty\right\} \text {. Then } \\
& \qquad \limsup _{t \rightarrow \infty} \frac{1}{t} \log |C(t)| \leq \rho_{0} \text { almost surely. }
\end{aligned}
$$

Proof See Appendix A.

This result covers many cases, some discussed previously by other authors:

- $C(t)$ independent and identically distributed (Dufresne, 1990; AEBI, et al., 1994);

- $C(t)$ an ergodic stochastic process and hence with $\mu(t)$ constant, giving $\rho_{0}=0$ (DUFRESNE, 1990);

- closed funds (for which $\mu(t)$ tends to zero in finite time) (PAPACHRISTOU and WATERS, 1991; PARKER, 1993, 1994a,b,c,d; FREES, 1990); 
- deterministic processes with exponentially bounded growth (for example, an office model with an assumed new business growth rate).

Theorem 4 gives a stronger condition for convergence than Lemma 3. For example, suppose $C(t)$ has a Log-Pareto distribution with drift: that is,

$$
\operatorname{Pr}[\log C(t)-\alpha t>x]=\frac{\text { constant }}{x^{\delta}} \text { for } x>x_{0} \text { and } \delta>0 .
$$

Then $E|C(t)|=\infty$ for all $t$ so that $\{C(t)\}$ does not satisfy the conditions for Theorem 4. Nevertheless, $\{C(t)\}$ does satisfy the conditions for Lemma 3, implying that $\lim \sup _{t \rightarrow \infty} t^{-1} \log |C(t)|$ is still less than infinity. Theorem 4 does, however, provide us with a condition for convergence which is often easier to verify, as will be demonstrated in the next section.

\section{A STOCHASTIC OFFICE MODEL}

We now develop the last of these examples to include office models in which cashflows are stochastic. The office's portfolio is assumed to consist only of policies which do not participate in the profits of the company. The model described includes stochastic mortality, stochastic growth of new business volumes, stochastic inflation in the size of individual policies and conditionally independent and identically distributed policy sizes at a given time of inception.

The generality of the model, here, means that the notation may appear to be quite heavy going, but the reader should concentrate on :

- the total premium income at time $t, P(t)$;

- the total benefit outgo at time $t, B(t)$, which consists of benefits payable on death during the year $(t-1, t]$, and on survival to duration $t$ for $t=1,2, \ldots$

Suppose that $\Lambda=\{\lambda(t)\}_{t=-\infty}^{\infty}, \lambda(t)=\left(\lambda_{1}(t), \lambda_{2}(t)\right)$, is the process with determines the volume of new business $\left(\lambda_{1}(t)\right)$ and the individual policy size index $\left(\lambda_{2}(t)\right)$.

Let $(x)_{t, j}$ represent the life corresponding to policy $j$ taken out at time $t$. All policyholders are aged $x$ at entry. Then, using standard notation, we have

${ }_{s} p_{x} \quad=$ probability that an individual now aged $x$ survives to age $x+s$

$=0$ for all $s>\omega-x$ for some limiting age $\omega<\infty$

and ${ }_{s-1} \mid q_{x}=$ the probability that an individual now aged $x$ dies between ages $x+s-1$ and $x+s$

$=0$ for all $s>\omega-x$

First we consider the total premium income at time $t$. This is

$$
\begin{aligned}
P(t) \quad & =\sum_{s=0}^{\infty} \sum_{j=1}^{N(t-s)} K_{s}^{P} A_{t-s}(j) I_{t-s, s}^{S}(j) \\
& =P_{1}(t)+P_{2}(t) \\
\text { where } P_{1}(t) & =\sum_{s=t}^{\infty} \sum_{j=1}^{N(t-s)} K_{s}^{P} A_{t-s}(j) I_{t-s, s}^{S}(j) \\
& =\begin{array}{l}
\text { premium income in respect of policies issued at or before } \\
\text { time } 0
\end{array}
\end{aligned}
$$




$$
\begin{aligned}
P_{2}(t) & \sum_{s=0}^{t-1} \sum_{j=1}^{N(t-s)} K_{s}^{P} A_{t-s}(j) I_{t-s, s}^{s}(j) \\
= & \text { premium income in respect of policies issued after time } 0 \\
= & \text { number of new policies taken out at time } s \\
\sim & P o\left(\lambda_{1}(s)\right),(s>0) \text { and, given } A, N(1), N(2), \ldots \text { are indepen- } \\
N(s) & \text { dent } \\
N(s) \mid \lambda_{1}(s) & \text { premium at duration } s \text { per unit of benefit } \\
= & \text { number of units of benefit for policy } j \text { taken out at time } t \\
K_{s}^{P} & \operatorname{Pr}\left(A_{t}(j) \leq x\right) \\
A_{t}(j) \quad & \text { with } A_{t}(1), A_{t}(2), \ldots \text { independent and identically distributed and } \\
F_{t}(x)= & \text { andependent of the } N(t) \\
& \text { and } A_{t}(j) \text { and } A_{s}(k) \text { are independent whenever } t \neq s \\
& \begin{cases}1 & \text { if }(x)_{t-s, j} \text { is still alive at time } t \\
0 \quad \text { otherwise }\end{cases}
\end{aligned}
$$

By leaving $K_{s}^{P}, K_{s}^{S}$ and $K_{s}^{D}$ (defined below) as essentially arbitrary functions of duration, we maintain a high degree of flexibility. In particular, this covers a wide variety of policy types including annuities and assurances.

Given these assumptions we can then say that $P_{2}(t) \mid A$ has the Compound Poisson distribution

$$
\begin{aligned}
& P_{2}(t) \mid \Lambda \sim \operatorname{CPo}\left(\Lambda_{P}(t), F_{t}^{P}\right) \\
& \text { where } \Lambda_{P}(t)=\sum_{s=0}^{t-1} \lambda_{1}(t-s)_{s} p_{x} \\
& \qquad F_{t}^{P}(x)=\frac{1}{\Lambda_{P}(t)} \sum_{s=0}^{t-1} \lambda_{1}(t-s)_{s} p_{x} F_{t-s}\left(x / K_{s}^{P}\right)
\end{aligned}
$$

If we assume that the history of the office is known up to and including time 0 then $P_{1}(t)$ is subject to much less uncertainty because the numbers and sizes of the existing policies are known. In any event $P_{1}(t)$ is equal to zero for all $t \geq$ $\omega-x$.

Turning now to the benefits process, $B(t)$, we can proceed in a similar, but slightly more complex, way:

$$
\begin{aligned}
B(t) & =\sum_{s=1}^{\infty} \sum_{j=1}^{N(t-s)}\left\{K_{s}^{S} A_{t-s}(j) I_{t-s, s}^{S}(j)+K_{s}^{D} A_{t-s}(j) I_{t-s, s}^{D}(j)\right\} \\
& =B_{1}(t)+B_{2}(t) \\
\text { where } B_{1}(t) & =\sum_{s=t}^{\infty} \sum_{j=1}^{N(t-s)}\left\{K_{s}^{S} A_{t-s}(j) I_{t-s, s}^{S}(j)+K_{s}^{D} A_{t-s}(j) I_{t-s, s}^{D}(j)\right\} \\
B_{2}(t) \quad & \sum_{s=1}^{t-1} \sum_{j=1}^{N(t-s)}\left\{K_{s}^{S} A_{t-s}(j) I_{t-s, s}^{S}(j)+K_{s}^{D} A_{t-s}(j) I_{t-s, s}^{D}(j)\right\}
\end{aligned}
$$


$K_{s}^{S} \quad=$ amount payable on survival to duration $s$ per unit benefit

$K_{s}^{D}$

$=$ amount payable on death during the year $(s-1, s]$ per unit benefit

$I_{t-s, s}^{D}(j) \quad= \begin{cases}1 & \text { if }(x)_{t-s, j} \text { dies during the year }(t-1, t] \\ 0 & \text { otherwise }\end{cases}$

and note that

$I_{t-s, s}^{S}(j)+I_{t-s, s}^{D}(j)= \begin{cases}1 & \text { if }(x)_{t-s, j} \text { is alive at } t-1 \\ 0 & \text { otherwise }\end{cases}$

As with the premium income we can then see that $B_{2}(t) \mid A$ has the Compound Poisson distribution

$B_{2}(t) \mid A \sim \operatorname{CPo}\left(\Lambda_{B}(t), F_{t}^{B}\right)$

where $A_{B}(t)=\sum_{s=1}^{t-1} \lambda_{1}(t-s)_{s-1} p_{s}$

$$
F_{t}^{B}(x)=\frac{1}{\Lambda_{B}(t)} \sum_{s=1}^{t-1} \lambda_{1}(t-s)\left\{{ }_{s} p_{x} F_{t-s}\left(x / K_{s}^{S}\right)+{ }_{s-1} \mid q_{x} F_{t-s}\left(x / K_{s}^{D}\right)\right\}
$$

We can also say that $B_{1}(t)=0$ for all $t>\omega-x$.

Now suppose that $m_{1}(t)=E\left[A_{t}(j)\right]$. Then for $t>\omega-x$

$$
\begin{aligned}
\mu(t) & =E(B(t)) \\
& =E[E(B(t) \mid A)] \\
& =E\left[\sum_{s=1}^{t-1} \lambda_{1}(t-s) m_{1}(t-s)\left(_{s} p_{x} K_{s}^{S}+{ }_{s-1} \mid q_{x} K_{s}^{D}\right)\right] \\
& =\sum_{s=1}^{(t)-t} E\left[\lambda_{1}(t-s) m_{1}(t-s)\right] k_{1}(s)
\end{aligned}
$$

where $k_{1}(s)={ }_{s} p_{x} K_{s}^{S}+{ }_{s-1} \mid q_{x} K_{s}^{D}$

To make further progress we need to make further assumptions about the claim size distributions and in the model for new business growth.

Suppose then we assume that $F_{t}(x)=F\left(x / \lambda_{2}(t)\right)$ : that is, $A_{t}(j) / \lambda_{2}(t)$ and $A_{s}(k) / \lambda_{2}(s)$ are identically distributed when $s \neq t$ and where $\lambda_{2}(t)$ represents the benefit inflation index. Then $m_{1}(t)=\lambda_{2}(t) m_{1}$.

$$
\Rightarrow \mu(t)=\sum_{s=1}^{(t)-x} m_{1} k_{1}(s) E\left[\lambda_{1}(t-s) \lambda_{2}(t-s)\right]
$$

Suppose also that

$$
\begin{aligned}
& \lambda_{1}(t)=\lambda_{1} \exp \left[\rho_{1} t+\sigma_{1} W_{1}(t)\right] \\
& \lambda_{2}(t)=\lambda_{2} \exp \left[\rho_{2} t+\sigma_{2} W_{2}(t)\right]
\end{aligned}
$$


where $W_{1}(t)$ and $W_{2}(t)$ are independent standard Brownian motions. In particular, they have the properties for $i=1,2$ :

$-W_{i}(0)=0$;

- if $t_{1}<t_{2}$ then $W_{i}\left(t_{2}\right)-W_{i}\left(t_{1}\right) \sim N\left(0, t_{2}-t_{1}\right)$;

- if $t_{1}<t_{2} \leq t_{3}<t_{4}$ then $W_{i}\left(t_{2}\right)-W_{i}\left(t_{1}\right)$ and $W_{i}\left(t_{4}\right)-W_{i}\left(t_{3}\right)$ are independent.

For $t<0 W_{1}(t)$ and $W_{2}(t)$ are known.

Then

$$
\begin{aligned}
E\left[\lambda_{1}(t) \lambda_{2}(t)\right] & =E\left[\lambda_{1}(t)\right] E\left[\lambda_{2}(t)\right] \\
& =\lambda_{1} \lambda_{2} \exp \left[\left(\rho_{1}+\rho_{2}+\frac{1}{2} \sigma_{1}^{2}+\frac{1}{2} \sigma_{2}^{2}\right) t\right] \\
& =\lambda_{1} \lambda_{2} \exp [\rho t] \\
\text { where } \rho & =\rho_{1}+\rho_{2}+\frac{1}{2} \sigma_{1}^{2}+\frac{1}{2} \sigma_{2}^{2}
\end{aligned}
$$

We therefore have

$$
\begin{aligned}
\mu(t) & =\lambda_{1} \lambda_{2} \sum_{s=1}^{\omega-x} m_{1} k_{1}(s) \exp [\rho(t-s)] \\
& =\lambda_{1} \lambda_{2} \exp (\rho t) \sum_{s=1}^{\omega-x} m_{1} k_{1}(s) \exp (-\rho s) \\
& =\mu \exp (\rho t) \\
\text { where } \mu & =\lambda_{1} \lambda_{2} \sum_{s=1}^{\omega-x} m_{1} k_{1}(s) \exp (-\rho s) .
\end{aligned}
$$

Hence, by Theorem 4 we deduce that

$$
\limsup _{t \rightarrow \infty} \frac{1}{t} \log |B(t)| \leq \rho \text { almost surely. }
$$

We can prove similarly that

$$
\limsup _{t \rightarrow \infty} \frac{1}{t} \log |P(t)| \leq \rho .
$$

Hence, if $\rho-\delta<0$ then, by Corollary 2

$$
Z(t)=\sum_{s=1}^{t} V(s) C(s)=\sum_{s=1}^{t} V(s) P(s)-\sum_{s=1}^{t} V(s) B(s)
$$

converges almost surely to some finite limit as $t$ tends to infinity. 


\section{DISCUSSION}

It is possible without great difficulty to relax many of the assumptions made in Section 5 .

- Here we assumed that all new entrants will be of the same age in an effort to contain the already complex notation. Relaxing this to include a spread of ages will result in a sum of conditionally independent Compound Poisson processes (given $A$ ) which is itself a Compound Poisson process.

- Similarly we could allow for more than one policy type, multiple state models (for example, permanent health insurance) and more than one risk group.

- Other forms of distribution for $N(t) \mid \lambda_{1}(t)$ would provide similar results. The Poisson assumption was made here for the convenience of its additive properties.

- Inclusion of expenses and reserves. (However, if we discount cashflows at the same rate of interest as that earned on the reserves, then the limiting value of $Z(t)$ will be unchanged.)

It should, therefore, follow that converge can be shown to occur for a wide range of office models, beyond the already general case described here.

Suppose that $N(t)=0$ for $t \leq 0$. Then, in the context of Section $5, \lim _{t \rightarrow \infty} Z(t)$ represents the present value of profit on future new business. The present paper has shown that, subject to certain conditions, this quantity is well defined and exists almost surely. It is a quantity which is of genuine practical interest since it allows actuaries to assess the underlying value of a company.

It is unlikely that the limiting distribution or the moments of $Z(t)$ will be known under the majority of circumstances. (However, where the cashflows in different years are independent, the methods of PARKER, 1993, 1994a,b,c can be used to find the distribution of $Z(t)$ for $t<\infty$. This then provides, for large $t$, an approximation to the limiting distribution of $Z(t)$.) It will often, therefore, be necessary to carry out a Monte-Carlo study, simulating sample paths of $Z(t)$. The results described in this paper indicate that $Z(t)$ will converge to its limit at least as fast as the deterministic annuity function $\bar{a}_{t}$ with force of interest $\delta-\rho$ tends to its limiting value. This gives us a useful guide as to when the difference between $Z(t)$ and its limit falls within the maximum tolerable level of error.

Some idea of the limiting distribution of $Z(t)$ can be obtained by applying the results of PAPACHRISTOU and WATERS (1991) and FREES (1990) for large portfolios. The analogue here is that the distribution of $\lambda_{1}^{-1} Z(t)$ tends to that of $\lambda_{1}^{-1} E\left[Z(t) \mid \delta(s), \lambda_{1}(s), \lambda_{2}(s),=1,2, \ldots, t\right]$ as $\lambda_{1}$ tends to infinity, and similarly the distribution of the limit of $Z(t)$ where this exists almost surely.

\section{ACKNOWLEDGEMENTS}

I would particularly like to thank an anonymous referee for helping me to improve Lemma 3 and Theorem 4 which led to a strengthening of the results contained in this paper. I would also like to thank Gary Parker, Angus Macdonald and Howard Waters for the helpful discussions they provided during the preparation of this paper. 
APPENDIX A

We make use of the following Corollary to Lemma 3.

\section{Corollary A.1}

If there exists a deterministic sequence a(t) converging to zero as $t$ tends to infinity such that $\sum_{t=1}^{\infty} \exp [-\rho t-t a(t)] E|c(t)|<\infty$ then

$\limsup _{t \rightarrow \infty} t^{-1} \log |C(t)| \leq \rho$ almost surely.

\section{Proof}

As in Lemma 3 let

$$
\begin{aligned}
E_{t} & =\left\{\frac{1}{t} \log |C(t)| \geq \rho+a(t)\right\} \\
& =\{|C(t)| \geq \exp [\rho t+\operatorname{ta}(t)]\}
\end{aligned}
$$

Now $E|C(t)| \geq c \operatorname{Pr}[|C(t)| \geq c]$ for any $c>0$ (for example, see Williams, 1991, Section 6.4). Hence

$$
\begin{gathered}
E|C(t)| \geq \exp [\rho t+t a(t)] \operatorname{Pr}\left(E_{l}\right) \\
\Rightarrow \sum_{t=1}^{\infty} \operatorname{Pr}\left(E_{t}\right) \leq \sum_{t=1}^{\infty} \exp [-\rho t-t a(t)] E|C(t)|<\infty
\end{gathered}
$$

This is the condition in Lemma 3.

\section{Proof of Theorem 4}

Take any $\rho_{2}>\rho_{0}$ and set $a(t)=0$ in Corollary A.1. Choose any $\rho_{1}$ such that $\rho_{0}<\rho_{1}<\rho_{2}$ and let $k=\sup \left\{\exp \left(-\rho_{1} t\right) \mu(t): t \geq 1\right\}$. Since $\rho_{1}>\rho_{0}, k$ must be finite. Then

$$
\begin{gathered}
\sum_{t=1}^{\infty} \exp \left[-\rho_{2} t-t a(t)\right] E|C(t)|=\sum_{t=1}^{\infty} \exp \left[-\rho_{1} t\right] E|C(t)| \exp \left[-\left(\rho_{2}-\rho_{1}\right) t\right] \\
<\sum_{t=1}^{\infty} k \exp \left[-\left(\rho_{2}-\rho_{1}\right) t\right]<\infty .
\end{gathered}
$$

Hence $\limsup _{t \rightarrow \infty} t^{-1} \log |C(t)| \leq \rho_{2}$ almost surely.

This is true for all $\rho_{2}>\rho_{0}$ so the result follows. 


\section{REFERENCES}

Aebı, M., Embrechts, P. and Mıкosch, T. (1994) Stochastic discounting, aggregate claims and the bootstrap. Advances in Applied Probability 26, 183-206.

Albrecht, P. (1985) A note on immunization under a general stochastic equilibrium model of the term structure. Insurance: Mathematics and Economics 4, 239-245.

BeEKMAN, J. A. and SHIU, E. S. W. (1988) Stochastic models for bond prices, function space integrals and immunization theory. Insurance: Mathematics and Economics 7, 163-173.

BOYLE, P.P. (1978) Immunization under stochastic models of the term structure. Journal of the Institute of Actuaries 105, 177-188.

BOYLE, P.P. (1980) Recent models of the term structure of interest rates with actuarial applications. 2/st International Congress of Actuaries, Zurich and Lausanne 4, 95-104.

BRANDT, A. (1986) The stochastic equation $Y_{n+1}=A_{n} Y_{n}+B_{n}$ with stationary coefficients. Advances in Applied Probability 18, 211 -220.

Brennan, M.J. and SChwARZ, E.S. (1979) A continuous time approach to the pricing of bonds. Journal of Banking and Finance 3, 135-155.

Cox, J.C., Ingtrsoll, J.E. and Ross, S. A. (1985) A theory of the term structure of interest rates. Econometrica 53, 385-407.

De Schepper, A., Tuenen, M. and Goovaerts, M. (1994) An analytical inversion of a Laplace transform related to annuities certain. Insurance: Mathematics and Economics 14, 33-37.

DHAENE, J. (1989) Stochastic interest rates and autoregressive integrated moving average processes. ASTIN Bulletin 19,131-138.

DhaEne, J. (1992) Actuarial functions and random rates of return. Bulletin of the Royal Society of Belgian Actuaries, 199/-92 85, 23-36.

DUfRESNE, D. (1990) The distribution of a perpetuity, with applications to risk theory and pension funding. Scandinavian Actuarial Journal 1-2, 39-79.

FreEs, E. W. (1990) Stochastic life contingencies with solvency considerations. Transactions of the Society of Actuctries 42, 91-148.

Heath, D., Jarrow, R. and Morton, A. (1990) Bond pricing and the term structure of interest rates: a discrete time approximation. Journal of Financial and Quantitative Analysis 25, 419-440.

HEATH, D., JaRROW, R. and MorTON, A. (1992) Bond pricing and the term structure of interest rates: a new methodology for contingent claims valuation. Econometrica 60, 77-105.

Karlin, S. and Taylor, H.M. (1975) A first course in stochastic processes: 2nd Edition. Academic Press, New York.

LONGSTAFF, F. A. and SChwART7, E.S. (1992) Interest rate volatility and the term structure: a two factor general equilibrium model. The Journal of Finance 47, 1259-1282.

Norberg, R. and Møller, C. M. (1994) Thiele's differential equation by stochastic interest of diffusion type. To appear in Scandinavian Actuarial Journal.

Papachristou, D. and Waters, H.R. (1991) Some remarks concerning interest rates in relation to long-term insurance policies. Scandinavian Actuarial Journal 2, 103-117.

Parker, G. (1993) Distribution of the present value of future cashflows. 3rd AFIR, Rome 2, 831-843.

PARKER, G. (1994a) Limiting distribution of the present value of a portfolio. ASTIN Bulletin 24, $47-60$.

Parker, G. (1994b) Stochastic analysis of an insurance portfolio. 4th AFIR, Orlando 1, 49-66.

PARker, G. (1994c) Moments of the present value of a portfolio of policies. Scandinavian Actuarial Journal 1, 53-67.

PARkER, G. (1994d) Two stochastic approaches for discounting actuarial functions. ASTIN Bulletin 24, $167-181$.

SERCU, P. (1991) Bond options and bond portfolio insurance. Insurance: Mathematics and Economics 10, 203-230.

VERVAAT, W. (1979) On a stochastic difference equation and a representation of non-negative infinitely divisible random variables. Advances in Applied Probability 11, 750-783.

WATER, H.R. (1978) The moments and distributions of actuarial functions. Journal of the Institute of Actuaries 105, 61-75.

WILKIE, A.D. (1986) A stochastic investment model for actuarial use. Transactions of the Faculty of Actuaries 39, 341-403.

WILKIE, A.D. (1987) Stochastic investment models - theory and practice. Insurance: Mathematics and Economics 6, 65-83. 
WILKIE, A.D. (1992) Stochastic investment models for XXIst century actuaries. 24th International Congress of Actuaries, Montreal 5, 119-137.

WILKIE, A. D. (1994) Stochastic modelling of long-term investment risks. To appear in the IMA Journal of Mathematics Applied in Business and Finance.

WilkiE, A.D. (1995) More on a stochastic asset model for actuarial use. To appear in British Actuarial Journal.

Williams, D. (1991) Probability with Martingales. CUP, Cambridge. 